\title{
Two cases of psychogenic anejaculation patients got normal ejaculation ability after penile vibratory stimulation or electroejaculation
}

\author{
Rong Cong, Qijie Zhang, Yi Wang, Xianghu Meng, Zengjun Wang, Ninghong Song \\ Department of Urology, the First Affiliated Hospital of Nanjing Medical University, Nanjing 210029, China \\ Correspondence to: Xianghu Meng; Ninghong Song. Department of Urology, the First Affiliated Hospital of Nanjing Medical University, Nanjing \\ 210029, China. Email: xhmeng888@163.com; songninghong_urol@163.com.
}

\begin{abstract}
Psychogenic anejaculation is a unique problem which accounts for $0.4-0.5 \%$ of anejaculation patients. Although patients with this problem may experience erections and nocturnal emissions (NEs), they do not experience ejaculation during either masturbation or coitus. The etiology is presently unknown. Penile vibratory stimulation (PVS) and electroejaculation (EEJ) have been the main methods of sperm retrieval for psychogenic anejaculation couples undergoing assisted reproduction technology (ART). However, there is no special treatment which can heal psychogenic anejaculation patients. Here, we report two psychogenic anejaculation patients who recovered normal ejaculation ability after PVS or EEJ procedure.
\end{abstract}

Keywords: Psychogenic anejaculation; penile vibratory stimulation (PVS); electroejaculation (EEJ); lumbar spinothalamic (LSt) cell

Submitted Jul 22, 2019. Accepted for publication Sep 29, 2019.

doi: $10.21037 /$ tau.2019.10.11

View this article at: http://dx.doi.org/10.21037/tau.2019.10.11

\section{Introduction}

Anejaculation is the complete absence of an antegrade or retrograde ejaculation despite an erection and is a rare cause of male infertility, accounting for $2 \%$ of all cases $(1,2)$. The cause of anejaculation is usually organic in nature such as spinal cord injury (SCI) $(3,4)$, retroperitoneal lymph node dissection (5), diabetes mellitus (6) and multiple sclerosis (7). However, if there is no cause, then it is considered psychogenic which is also known as idiopathic.

Psychogenic anejaculation is a unique problem which accounts for $0.4-0.5 \%$ of anejaculation patients (2). Although patients with this problem may experience erections and nocturnal emissions (NEs), they do not experience ejaculation during either masturbation or coitus (8-12). The etiology is presently unknown, but according to earlier papers, it may be associated with psychological factors such as cultural or religious beliefs, fear of inducing pregnancy, or emotional distress. Penile vibratory stimulation (PVS) and electroejaculation (EEJ) have been the main methods of sperm retrieval for psychogenic anejaculation couples undergoing assisted reproduction technology (ART). However, there is still no special treatment which can heal psychogenic anejaculation patients. Here, we report two psychogenic anejaculation patients who recovered normal ejaculation ability after PVS or EEJ procedure.

\section{Case presentation}

We present two patients referred to our department for anejaculation. Both had normal libido and had intercourse with their girlfriend or wife about twice a week. They never ejaculated during either coitus or masturbation. They experienced NEs on an average of three to five times per month after puberty. Physical examination of the men showed normal genitalia and no signs of endocrinological or neurological abnormalities. The levels of testosterone, 
follicle-stimulation hormone, luteinizing hormone, and prolactin were within the normal range. Retrograde ejaculation was ruled out by postcoital urine analysis. According to their medical history, the diagnosis of psychogenic anejaculation was established. The two patients selected PVS or EEJ to retrieve their semen for purposes of ART, respectively.

\section{Case 1}

A healthy 25-year-old male was diagnosed with psychogenic anejaculation at our department. He worked as an office clerk and had a girlfriend for 3 years, but they parted later due to the anejaculation.

A trial for a PVS procedure as described previously (13) was offered as the first-line treatment which led to his first ejaculation while awake in $30 \mathrm{~min}$ resulting in an ejaculate of $2.0 \mathrm{~mL}$ with a concentration of $532.78 \times 10^{6}$ spermatozoa/ $\mathrm{mL}$ and a poor progressive sperm motility of $1.42 \%$. Because of the poor semen quality, he did a second PVS procedure 5 days later which also succeeded after $30 \mathrm{~min}$ resulting in an ejaculate of $3.8 \mathrm{~mL}$ with a concentration of $243.23 \times 10^{6}$ spermatozoa/mL and a progressive sperm motility of $26.93 \%$. Four days later, he told us that he could ejaculate by masturbation. We then asked him to masturbate in the hospital which succeeded in about $5 \mathrm{~min}$ resulting in an ejaculate of $1.8 \mathrm{~mL}$ with a concentration of $344.36 \times 10^{6}$ spermatozoa/mL and a progressive sperm motility of $60.08 \%$. Because he did not have a girlfriend at that time, we did not know whether he could ejaculate by coitus. With close regular follow-up, he told us that he could ejaculate with his second girlfriend by coitus. At the time writing, he could ejaculate by masturbation and coitus.

\section{Case 2}

A healthy 26-year-old male with his 25-year-old wife was referred to our department for infertility secondary to psychogenic anejaculation. He had been married for 5 years. The 25-year-old wife had regular menstrual cycles. Her general and gynecological examination was normal.

Unlike case 1, the couples selected EEJ to retrieve their semen for its high success rate. The EEJ procedure, as described previously (13), was performed under general anesthesia which resulted in an ejaculate of about $0.3 \mathrm{~mL}$ with a concentration of $7.78 \times 10^{6}$ spermatozoa $/ \mathrm{mL}$ and no progressive sperm. We asked him to do another EEJ after 2 weeks. However, after 1 week, he told us that he could ejaculate through masturbation and coitus with his wife. We then asked him to masturbate in the hospital, which succeeded in about 5 mins resulting in an ejaculate of $1.0 \mathrm{~mL}$ with a concentration of $54.47 \times 10^{6}$ spermatozoa $/ \mathrm{mL}$ and a progressive sperm motility of $33.19 \%$. With close regular follow-up, he told us that his wife finally became pregnant and gave birth to a healthy baby.

\section{Discussion}

There is no special treatment for psychogenic anejaculation patients. Most cases require medical assistance. PVS and EEJ methods have been the main methods of sperm retrieval for psychogenic anejaculation couples undergoing ART. Nowadays, studies only focus on the semen quality and pregnancy rate of different semen retrieve methods for psychogenic anejaculation (8-12). However, there is little information about what happens to these psychogenic anejaculation patients after PVS or EEJ procedure.

As we all know, ejaculation is a physiological process controlled by spinal and supraspinal sites (brainstem and hypothalamus) which consists of two phases, emission (secretion and movement of seminal fluids to the urethra) and expulsion (forceful ejection of urethral contents). Failure in the initial step leads to anejaculation (14).

According to our two psychogenic anejaculation cases, one patient was treated by PVS while awake. Meanwhile, the other patient was treated by EEJ under general anesthesia; however, both were able to achieve normal ejaculation ability and did not receive any other treatments. So, we hypothesize this may be related to the regulation of the spinal and not supraspinal sites.

Lumbar spinothalamic (LSt) cells, also known as spinal ejaculation generators, were first discovered by Truitt and Coolen within the central gray area of lumbar segments L3L4 which play a pivotal role in generation of ejaculation (15). They receive stimulation related to onset of ejaculation and, in turn, trigger the ejaculatory reflex. LSt cells can coordinate sympathetic, parasympathetic, and motor outflow to induce the two phases of ejaculation $(15,16)$.

Allard et al. hypothesized that rapid ejaculation might be related to hypersensitivity of LSt cells (17). In other words, anejaculation might be due to a hyposensitivity of LSt cells. In our study, the recovery of the two patients' ejaculation ability after strong stimulation might be related to the activation of LSt cells. Hence, psychogenic anejaculation may be related to the dysfunction of LSt cells. And this may be why psychotherapies are not effective in these patients. 
There may be several reasons for why there are no studies reporting this phenomenon. Firstly, the incidence of psychogenic anejaculation is exceptionally low, which has not attracted the attention of researchers. The second reason is that all published studies only focus on the semen quality and pregnancy rate of different semen retrieval methods for psychogenic anejaculation. There is little information about what happens to these patients after semen retrieval. The third reason may be related to the abstinence of psychogenic anejaculation patients when their partners become pregnant after ART.

Also, there were limitations to our study. First, it was a case report and retrospective study. There still need further and large-scale investigations. Second, we could not identify the exact mechanism that would explain why the two patients could recover normal ejaculation ability after PVS or EEJ procedures while other psychogenic anejaculation patients could not.

In summary, our work suggests that PVS and EEJ are not only just methods to retrieve semen samples but can also heal certain psychogenic anejaculation patients. These findings may have potential significance for encouraging urologists and andrologists to study the etiology of psychogenic anejaculation further.

\section{Acknowledgments}

None.

\section{Footnote}

Conflicts of Interest: The authors have no conflicts of interest to declare.

Ethical Statement: The authors are accountable for all aspects of the work in ensuring that questions related to the accuracy or integrity of any part of the work are appropriately investigated and resolved. Written informed consent was obtained from the patient for publication of this Case Report.

\section{References}

1. Colpi G, Weidner W, Jungwirth A, et al. EAU guidelines on ejaculatory dysfunction. Eur Urol 2004;46:555-8.

2. Soeterik TF, Veenboer PW, Lock TM. Electroejaculation in psychogenic anejaculation. Fertil Steril 2014;101:1604-8.
3. Heruti RJ, Katz H, Menashe Y, et al. Treatment of male infertility due to spinal cord injury using rectal probe electroejaculation: the Israeli experience. Spinal Cord 2001;39:168-75.

4. Brackett NL, Ibrahim E, Iremashvili V, et al. Treatment for ejaculatory dysfunction in men with spinal cord injury: an 18-year single center experience. J Urol 2010;183:2304-8.

5. Hsiao W, Deveci S, Mulhall JP. Outcomes of the management of post-chemotherapy retroperitoneal lymph node dissection-associated anejaculation. BJU Int 2012;110:1196-200.

6. Gerig NE, Meacham RB, Ohl DA. Use of electroejaculation in the treatment of ejaculatory failure secondary to diabetes mellitus. Urology 1997;49:239-42.

7. Fode M, Krogh-Jespersen S, Brackett NL, et al. Male sexual dysfunction and infertility associated with neurological disorders. Asian J Androl 2012;14:61-8.

8. Everaert K, Oosterlinck W. Diagnosis and treatment of psychosocial induced anejaculation or anorgasm by vibratory stimulation. Acta Urol Belg 1997;65:59-61.

9. Hovav Y, Kafka I, Dan-Goor M, et al. Electroejaculation in combination with intracytoplasmic sperm injection in patients with psychogenic anejaculation results in lower fertilization rates. Fertil Steril 1998;69:561-3.

10. Hovav Y, Yaffe H, Zentner B, et al. The use of ICSI with fresh and cryopreserved electroejaculates from psychogenic anejaculatory men. Hum Reprod 2002;17:390-2.

11. Gat I, Maman E, Yerushalmi G, et al. Electroejaculation combined with intracytoplasmic sperm injection in patients with psychogenic anejaculation yields comparable results to patients with spinal cord injuries. Fertil Steril 2012;97:1056-60.

12. Meng X, Fan L, Wang T, et al. Electroejaculation combined with assisted reproductive technology in psychogenic anejaculation patients refractory to penile vibratory stimulation. Transl Androl Urol 2018;7:S17-22.

13. Meng X, Fan L, Liu J, et al. Fresh semen quality in ejaculates produced by nocturnal emission in men with idiopathic anejaculation. Fertil Steril 2013;100:1248-52.

14. Coolen LM, Allard J, Truitt WA, et al. Central regulation of ejaculation. Physiol Behav 2004;83:203-15.

15. Truitt WA, Coolen LM. Identification of a potential 
ejaculation generator in the spinal cord. Science 2002;297:1566-9.

16. Staudt MD, Truitt WA, McKenna KE, et al. A pivotal role of lumbar spinothalamic cells in the regulation

Cite this article as: Cong R, Zhang Q, Wang Y, Meng X, Wang Z, Song N. Two cases of psychogenic anejaculation patients got normal ejaculation ability after penile vibratory stimulation or electroejaculation. Transl Androl Urol 2019;8(6):758-761. doi: 10.21037/tau.2019.10.11 of ejaculation via intraspinal connections. J Sex Med 2012;9:2256-65.

17. Allard J, Truitt WA, McKenna KE, et al. Spinal cord control of ejaculation. World J Urol 2005;23:119-26. 